Original article

https://www.journal-imab-bg.org
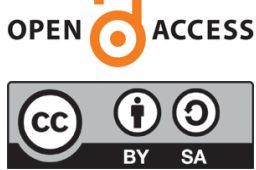

\title{
ELEVATION OF THE CENTER OF ROTATION WITH THE USE OF JUMBO CUPS IN REVISION TOTAL HIP ARTHROPLASTY - A RADIO- GRAPHIC STUDY
}

\author{
Maksim Zagorov ${ }^{1}$, Kalin Mihov ${ }^{1}$, Svetoslav Dobrilov ${ }^{1}$, Gergana Nenova ${ }^{2}$ \\ 1) Department of Orthopaedics and Traumatology, University Hospital St. Ma- \\ rina - Varna, Bulgaria. \\ 2) Training sector of Kinesitherapy, Faculty of Public Health, Medical Univer- \\ sity, Varna, Bulgaria.
}

\section{SUMMARY:}

Background: Acetabular revision with extra-large (jumbo) cementless cups is an effective treatment for many cavitary and segmental peripheral bone defects. However, hip center elevation may occur with the use of a jumbo cup owing to the superior direction of reaming and the increased diameter of the component compared with the native acetabulum.

Purposes: The primary goal of this study was to evaluate the radiographically observed hip center elevation with the use of jumbo cups in acetabular and total hip revision at our institution.

Materials and methods: We retrospectively reviewed control radiographic images of 43 consecutive patients treated with acetabular and total hip revision arthroplasty with a press-fit cementless cup. The difference between the height of the rotation center of the acetabular cup and the height of the rotation center of the contralateral native hip relative to the inter teardrop line was radiographically measured.

Results: The radiographically measured mean elevation of the rotation center of the jumbo cup was $8,75 \mathrm{~mm}$, which yielded an average increase in the height of the rotation centre of 83,6. A mean vertical shift of the rotation center of $0,72 \mathrm{~mm}$ was estimated for every $1 \mathrm{~mm}$ increase in the size of the jumbo cup.

Conclusions: Our results indicate that the use of jumbo cups in revision hip arthroplasty leads to elevation of the rotation center of the hip with an average of $9 \mathrm{~mm}$. This could be considered by the surgeon in an attempt to restore leg length, stability and biomechanics of the prosthetic hip.

Keywords: hip revision, jumbo cup, center of rotation, elevation,

\section{INTRODUCTION:}

Acetabular revision with extra-large (jumbo) cementless cups is an effective treatment for many cavitary and segmental peripheral bone defects demonstrating reliable fixation and good long term survivorship [1, 2, 3]. With the availability of highly porous trabecular metal cups and augments the presence of at least $50 \%$ contact of the cup with vital host bone seem to be of less importance and according to some the only contraindication for implementation of this technique alone is pelvic discontinuity [4, 5]. Despite the lack of commonly accepted definition the term jumbo cup usually refers to components of diameter greater than $66 \mathrm{~mm}$ in men or $62 \mathrm{~mm}$ in women or approximately $10 \mathrm{~mm}$ larger than the native contralateral acetabulum $[6,7]$. The goal of the technique is to fill the acetabulum from the ilium to the 'tear drop' with the component after creating a new hemispherical acetabular bone bed both antero-posteriorly and supero-inferiorly [8]. (fig. 1)

Fig. 1. Preoperative aseptic cup loosening with migration and bone osteolysis and the postoperative result after implantation of "jumbo cup"

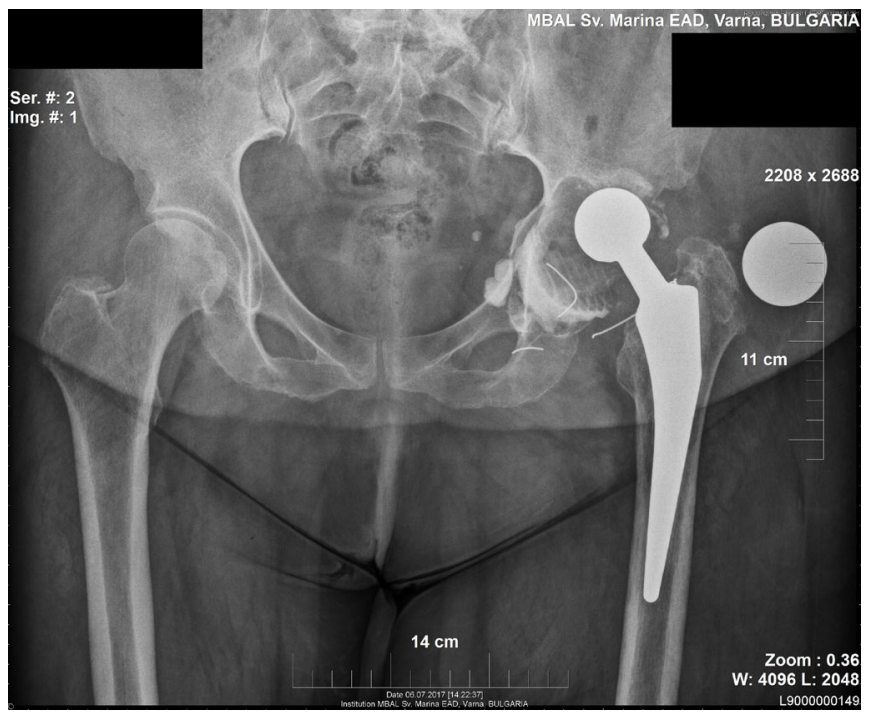




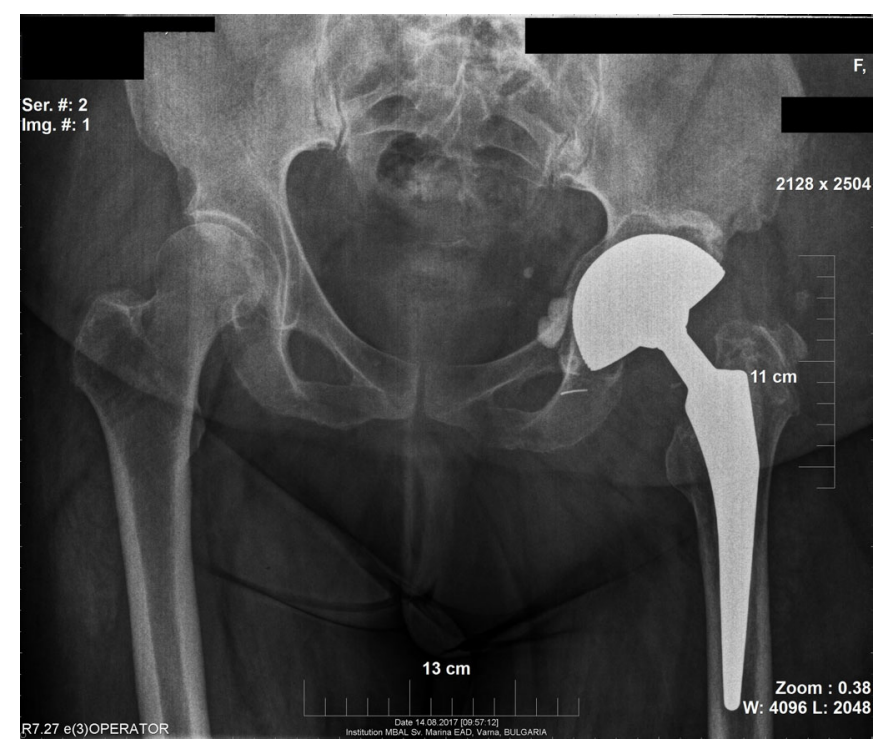

Jumbo cups offer several advantages over alternative strategies of acetabular revision. This method allows for the implementation of standard surgical technique with the use of hemispherical reamers and screw transfixation. The large contact area between the porous implant and the host bone facilitates biological fixation and strain distribution over a large area of the pelvis. Furthermore, the large cup itself is used to fill various sizes of bone defects, thus potentially reducing the need for allografts and augments [9]. However, the preparation of the acetabular surface to accept an implant that is much larger than the native acetabulum requires reaming into both anterior and posterior columns and medial wall of the acetabulum with a certain amount of bone loss. Furthermore, hip center elevation may occur with the use of a jumbo cup owing to the superior direction of reaming and the increased diameter of the component compared with the native acetabulum [10]. Elevation of the hip center of rotation has been associated with altered hip biomechanics, dislocation and instability, leg-lenth discrepancy and suboptimal clinical results [11]. In order to minimize hip center elevation in acetabular revision with a jumbo cup, we use the recommended technique of sequential reaming in contact with the inferior edge of the acetabulum which enables implanting the cup in a relatively anatomic position [12].

The primary goal of this study was to evaluate the radiographically observed hip center elevation with the use of jumbo cups in acetabular and total hip revision at our institution. Our secondary goal was to compare the amount of hip center elevation between a group of acetabular and total hip revisions with the use of jumbo cups and a control group of acetabular and total hip revisions using a cementless pressfit cup of a size smaller than the jumbo size criteria.

\section{MATERIALS AND METHODS:}

43 consecutive acetabular and total hip revision arthroplasties, performed at our department between January 2014 and June 2018, were retrospectively reviewed. Of those 28 patients with a native contralateral hip joint were identified. A titanium hemispheric cup with HA coating was used in 9 patients and a highly porous tantalum hemispheric cup was used in 1 case. In the remaining 18 cases, a cementless spherocylindrical stainless steel dual mobility cup with double layer coating of porous titanium and HA was applied. Additional screw fixation was used as necessary. In 12 cases (5 men and 7 women) the acetabular components used were defined as jumbo cups (outer diameter larger than $66 \mathrm{~mm}$ in men and 62 in women or difference $\geq 10 \mathrm{~mm}$ with the contralateral native hip) and in the rest 16 cases ( 8 men and 8 women) a cementless pressfit cup of a size smaller than the jumbo size criteria was used. Thus, two groups were defined: a jumbo cup group (JC) and a normal size cup group (NSC). In both groups, a radiographic analysis of the control AP X-ray images at the 6 th postoperative week using a software for 2 dimensional preoperative planning (Medicad 2,20; Hectec, Germany) was performed. The difference between the height of the rotation center of the acetabular cup and the height of the rotation center of the contralateral native hip relative to the interteardrop line was measured. This was considered as the amount of vertical shift of the center of rotation of the prosthesis. (Fig. 2)

Fig. 2. Postoperative measurement of the elevation of the center of rotation in a series of patients with jumbo cups.
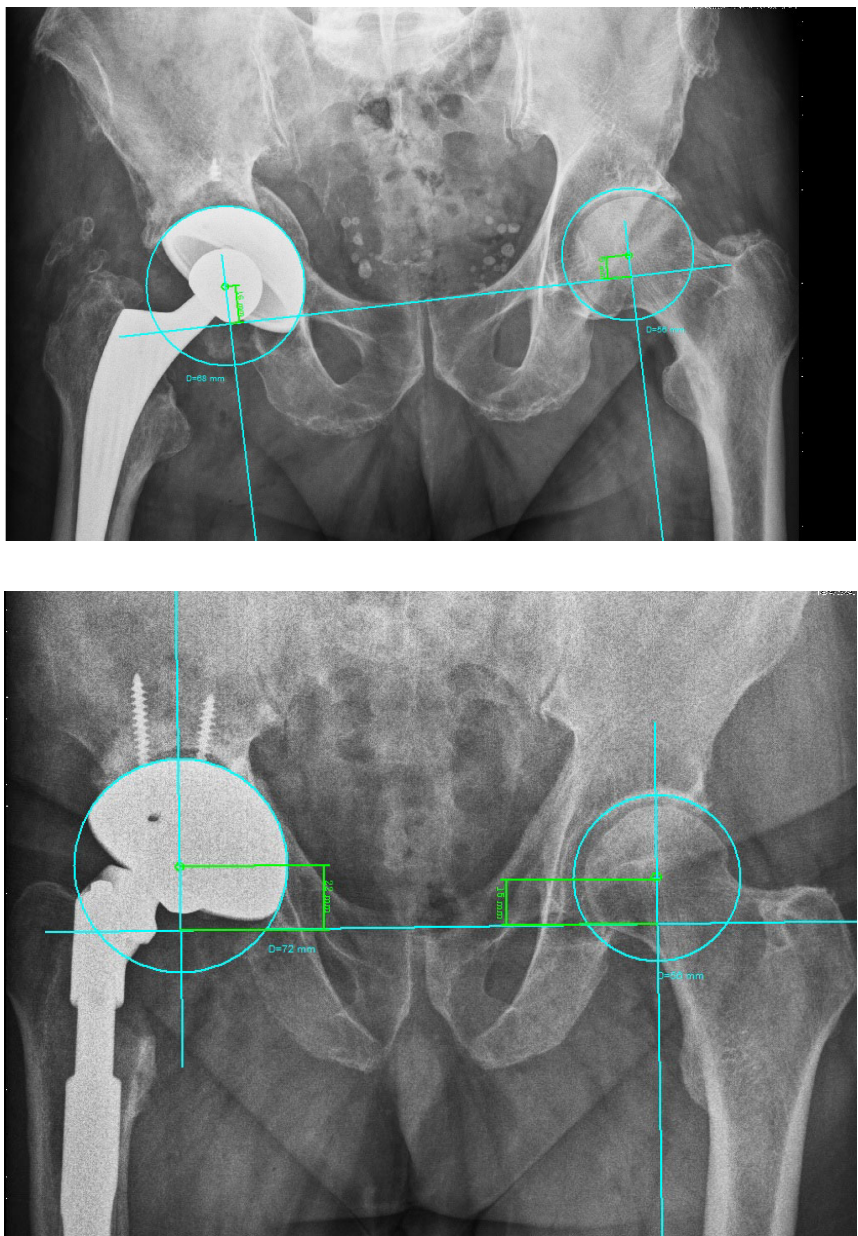


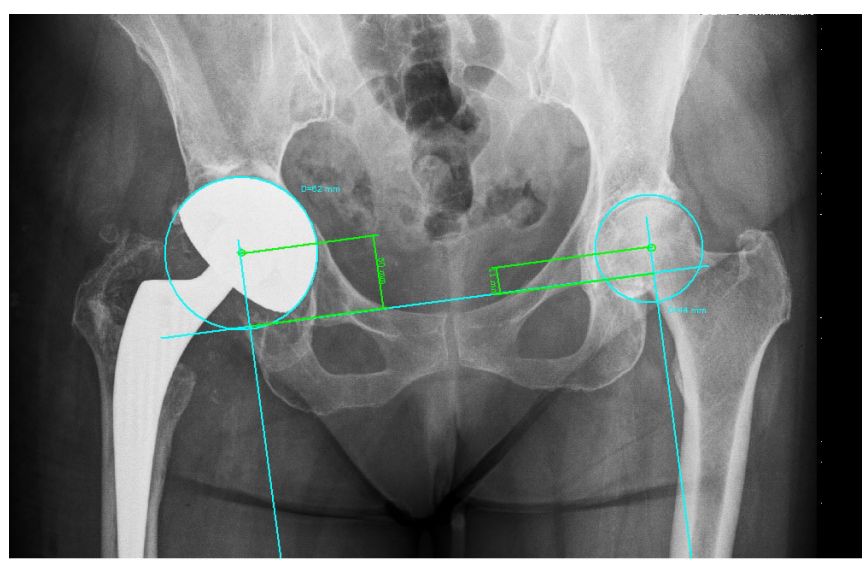

Elevation of the rotation center was compared between the two groups and between males and females in the jumbo cup group with independent samples t-test.

After calibrating all images according to the magnification ball or the previously known size of the implanted cup the centres of rotation of both prosthetic and the native hip were determined by drawing a circle best aligned with the margins of the acetabular cup or the native acetabulum. The height of the hip center was estimated by measuring the length of a perpendicular line from the interteardrop line to the hip rotation center. This was done for the acetabular cup and the contralateral native acetabulum and the difference between the vertical height of both hip centres was the measure of the rotation center elevation. The same process was repeated in both the JC group and the NSC group.

\section{RESULTS:}

Mean age was 70,83 years $(\mathrm{SD} 6,41 ; 57-77)$ in the JC group and 66 years (SD 12,72; 37-77) in the NSC group respectively. The mean outer diameter of the cup in the JC group was $64,5 \mathrm{~mm}(68,6 \mathrm{~mm}$ in men and $61,6 \mathrm{~mm}$ in women) (SD 4,34; 57-72) and the mean difference with the size of the native contralateral acetabulum was 12,1 $\mathrm{mm}$ (11,8 $\mathrm{mm}$ in men and 12,3 $\mathrm{mm}$ in women) (SD 2,35; $9-16)$. The radiographically measured mean elevation of the rotation center of the jumbo cup was $8,75 \mathrm{~mm}(9,2$ $\mathrm{mm}$ in men and $8,43 \mathrm{~mm}$ in women) (SD 5,50;2-19), which yielded an average increase in the height of the rotation centre of $83,5 \%$ (98\% in men and $73,2 \%$ in women) (SD 56,$17 ; 15,4-172)$. A mean vertical shift of the rotation center of $0,73 \mathrm{~mm}(0,77 \mathrm{~mm}$ in men and $0,7 \mathrm{~mm}$ in women) (SD 0,38; 0,2-1.26) was estimated for every $1 \mathrm{~mm}$ increase in the size of the jumbo cup.

The mean outer diameter of the cup in the NSC group was $57,7 \mathrm{~mm}(60,6 \mathrm{~mm}$ in men and $54,8 \mathrm{~mm}$ in women) (SD 3,59; 51-61) and the mean difference with the size of the native contralateral acetabulum was 4,4 $\mathrm{mm}(4,5 \mathrm{~mm}$ in men and 4,25 $\mathrm{mm}$ in women) (SD 1,15; 3 $7)$. The radiographically measured mean elevation of the rotation center of the normal-sized cup was $3,3 \mathrm{~mm}(3,6$ $\mathrm{mm}$ in men and $3 \mathrm{~mm}$ in women) (SD 1,78; 1-6), which yielded an average increase in the height of the rotation centre of $22,7 \%(23,5 \%$ in men and $28,24 \%$ in women) (SD 11,$74 ; 5,6-46,2)$. A mean vertical shift of the rotation center of $0,71 \mathrm{~mm}(0,82 \mathrm{~mm}$ in men and $0,59 \mathrm{~mm}$ in women) (SD 0,5; 0,2-2) was estimated for every $1 \mathrm{~mm}$ increase in the size of the jumbo cup.

There was a significant difference in the outer cup diameter $(p=0,000)$ and the difference with the size of the native contralateral acetabulum $(p=0,007)$ between the JC and the NSC group. Mean elevation of the center of rotation in the JC group was higher than the elevation of the center of rotation in the NSC group and the difference was statistically significant ( $\mathrm{p}=0,001$ at $95 \% \mathrm{CI}$ ). The amount of elevation of the center of rotation did not differ significantly between men and women in the jumbo cup group. $(p=0,823)$. The mean vertical shift of the rotation for every $1 \mathrm{~mm}$ increase in the size of the implanted cup did not differ significantly between the two groups $(p=0,895)$.

\section{DISCUSSION:}

Jumbo press fit acetabular cups are commonly used to address extensive bone defects in revision arthroplasty of the acetabulum with a survivorship rate of $90 \%$ and $85 \%$ at 10 and 20 years respectively [2, 3]. However, both reaming in a superior direction with hemispherical preparation of the acetabular bony surface and implantation of a cup much larger, than the size of the native acetabulum can lead to elevation of the rotation center. We estimated the amount of radiographically observed elevation in the hip center with the use of jumbo cups and how this compared to the elevation of the center of rotation in a group with normal size cup revision arthroplasty.

Our radiographic analysis demonstrated mean elevation of the rotation center in the jumbo cup group of $8,75 \mathrm{~mm}$ despite the implementation of surgical technique that aims to place the cup in contact with the inferior edge of the acetabulum. Hip center elevation in the JC group was larger in men than in women but the difference was not statistically significant. There was a statistically significant higher elevation of the rotation centre in the jumbo cup group compared to the normal-sized cup revision group. Furthermore, the amount of vertical shift of the rotation center for every $1 \mathrm{~mm}$ increase in the outer diameter of the cup was almost equal in both groups $(0,73$ $\mathrm{mm}$ vs $0,71 \mathrm{~mm}$ ). Assuming that the same surgical technique with implanting the acetabular cup as anatomically as possible was applied in every case in both hip revision groups we suggest that the growing diameter of the jumbo cup is mainly responsible for the observed elevation of the prosthetic center of rotation.

In a computer simulation study based on 265 tridimensional pelvic models, Nwankwo et al. demonstrated that the hip rotational center shifted $0.27 \mathrm{~mm}$ superiorly and $0.02 \mathrm{~mm}$ anteriorly for every $1-\mathrm{mm}$ increase in reamer diameter (10). In a subsequent clinical study, the same authors reported a mean radiographic elevation of the center of rotation of $11 \mathrm{~mm}$ relative to the contralateral hip in a series of 98 revision hip arthroplasties with jumbo cups [10].

Our data is similar to the results of this study. The slight difference in the mean hip centre elevation is prob- 
ably due to the smaller mean size of the jumbo cups in our series $(64,5 \mathrm{~mm}$ vs $67,3 \mathrm{~mm})$. A retrospective analysis of 79 total hip arthroplasty revisions by Dou et al., reported that the acetabular side significantly contributes to hip center elevation and leg length shortening after revision total hip arthroplasty [11].

In a biomechanical study, Delp et al. showed that superolateral placement of the hip center $(2 \mathrm{~cm}$ superior and $2 \mathrm{~cm}$ lateral) decreases the moment arm of the hip abductors by an average of $28 \%$ which can not be compensated by a longer femoral head. Of notice, the isolated proximal shift of the hip center compensated by a longer femoral head reduces the force generating capacity of the hip abductors by less than $10 \%$ [12]. We estimated a mean proximalisation of the prosthetic rotation center of 0,73 $\mathrm{mm}$ for every $1 \mathrm{~mm}$ increase in the outer diameter of the jumbo cup, relative to the contralateral native hip center. This could give the surgeon an intraoperative idea of the compensatory elongation needed at the femoral side. Dislocation is the most frequent complication after revision hip arthroplasty with a jumbo acetabular component ranging from $4,2 \%$ to $12 \%$ in large series.[1-3, 6]

Together with the extensive surgical approach and the mismatch of the sizes of the acetabular component and the femoral head the elevation of the rotation center in jumbo cup revision hip arthroplasty may be one of the multiple factors that contribute to soft tissue laxity and hip instability. The negative effect of the elevation of the rotation center on the hip offset and leg length can potentially be compensated for by the geometry and placement of the femoral component and the length of the prosthetic head (10).

Furthermore, dislocation risk in revision total hip arthroplasty can be decreased with the use of large diameter femoral heads or dual-mobility acetabular cups. The latter demonstrated superiority over $40 \mathrm{~mm}$ femoral heads in terms of dislocation and re-revision rate in a recent comparative study by Hartzler et al. [15, 16]. A potential disadvantage of the monoblock pressfit cementless dual mobility cups is the inability both to visualise the acetabular floor during impaction and to use transfixation screws which may compromise primary fixation in the setting of bony defects. Despite that our experience with jumbo-sized dual-mobility cementless cups show that, meticulous surgical technique with achievement of satisfactory equatorial pressfit in the acetabular rim can result in good primary stability of the metal back in the majority of cases. In the presence of severe segmental acetabular defects, a dual-mobility cup can be cemented in a highly-porous cementless hemispheric cup of jumbo size [17].

This study has several limitations. First, it is the small number of patients included. Secondly, neither intraobserver nor interobserver repeatability testing were included in the study as all measurements were done only once per case by one of us (M.Z.). Third, measurement errors can potentially occur in a radiographic study despite the use of digital software and standardized patient positioning. Finally, the amount of elevation of the center of rotation of the cup resulting from proximal implantation of the cup relative to the lower acetabular edge was not separately measured but, all surgeries in both groups were performed by the same team using the same surgical technique.

\section{CONCLUSION:}

Our results indicate that the use of jumbo cups in revision hip arthroplasty leads to elevation of the rotation center of the hip with an average of $9 \mathrm{~mm}$. This could be considered by the surgeon in an attempt to restore leg length, stability and biomechanics of the prosthetic hip.

\section{REFERENCES:}

1. Gustke KA, Levering MF, Miranda MA. Use of jumbo cups for revision of acetabulae with large bony defects. J Arthroplasty. 2014 Jan;29(1):199-203. [PubMed] [Crossref]

2. Lachiewicz PF, Soileau ES. Fixation, survival, and dislocation of jumbo acetabular components in revision hip arthroplasty. J Bone Joint Surg [Am]. 2013 Mar 20;95(6):543-8. [PubMed] [Crossref]

3. von Roth P, Abdel MP, Harmsen WS, Berry DJ. Uncemented jumbo cups for revision total hip arthroplasty: a concise follow-up, at a mean of twenty years, of a previous report. J Bone Joint Surg [Am]. 2015 Feb 18; 97(4):284-7. [PubMed] [Crossref]

4. Lakstein D, Backstein D, Safir
O, Kosashvili Y, Gross AE. Trabecular Metal cups for acetabular defects with $50 \%$ or less host bone contact. Clin Orthop Relat Res. 2009 Sep;467(9): 2318-24. [PubMed] [Crossref]

5. Lachiewicz PF, Soileau ES. Tantalum components in difficult acetabular revisions. Clin Orthop Relat Res. 2010 Feb;468(2):454-8. [PubMed] [Crossref]

6. Whaley AL, Berry DJ, Harmsen WS. Extra-large uncemented hemispherical acetabular components for revision total hip arthroplasty. J Bone Joint Surg Am. 2001 Sep;83(9):13527. [PubMed] [ $\underline{\text { Crossref] }}$

7. Patel JV, Masonis JL, Bourne $\mathrm{RB}$, Rorabeck CH. The fate of cementless jumbo cups in revision hip arthroplasty. J Arthroplasty 2003;

\section{8(2):129. [PubMed] [Crossref]}

8. Lachiewicz PF, Watters TS. The jumbo acetabular component for acetabular revision. Bone Joint J. 2016 Jan;98-B(1 Suppl A):64-7. [PubMed] [ Crossref]

9. Gustke KA. Jumbo cup or high hip center: is bigger better? J Arthroplasty. 2004 Jun;19(4 Suppl 1):1203. [PubMed] [Crossref]

10. Nwankwo C, Dong NN, Heffernan CD, Ries MD. Do jumbo cups cause hip center elevation in revision THA? A computer simulation. Clin Orthop Relat Res. 2014 Feb; 472(2): 572-576. [P [Crossref]

11. Dou Y, Zhou Y, Tang Q, Yang D, Liu J. Leg-length discrepancy after revision hip arthroplasty: are 
modular stems superior? J Arthroplasty. 2013 Apr;28(4):676-9. [PubMed] [ Crossref]

12. Delp SL, Wixson RL, Komattu $\mathrm{AV}$, Kocmond JH. How superior placement of the joint center in hip arthroplasty affects the abductor muscles. Clin Orthop Relat Res. 1996 Jul; (328):137-46. [PubMed] [Crossref]

13. Tanzer M. Role and results of the high hip center. Orthop Clin North Am. 1998 Apr;29(2):241-7. [PubMed] [Crossref]

14. Nwankwo CD, Ries MD. Do jumbo cups cause hip center eleva- tion in revision THA? A radiographic evaluation. Clin Orthop Relat Res. 2014 Sep; 472(9): 2793-2798 [PubMed] [Crossref]

15. Garbuz DS, Masri BA, Duncan CP, Greidanus N, Bohm E, Petrak M, et al. The Frank Stinchfield Award: Dislocation in revision THA: do large heads (36 and $40 \mathrm{~mm}$ ) result in reduced dislocation rates in a randomized clinical trial? Clin Orthop Relat Res. 2012 Feb;470(2):351-6. [PubMed] [ $\underline{\text { Crossref] }}$

16. Hartzler MA, Abdel MP,
Sculco PK, Taunton MJ, Pagnano MW, Hanssen AD. Otto Aufranc Award: Dual-mobility Constructs in Revision THA Reduced Dislocation, Rerevision, and Reoperation Compared With Large Femoral Heads. Clin Orthop Relat Res. 2018 Feb;476(2): 293-301. [PubMed] [ Crossref]

17. Brüggemann A, Mallmin H, Hailer NP. Do dual-mobility cups cemented into porous tantalum shells reduce the risk of dislocation after revision surgery? Acta Orthop. 2018 Apr;89(2):156-162. [PubMed]

Please cite this article as: Zagorov M, Mihov K, Dobrilov S, Nenova G. Elevation of the center of rotation with the use of jumbo cups in revision total hip arthroplasty - a radiographic study. J of IMAB. 2021 Jan-Mar;27(1):3518-3522.

DOI: https://doi.org/10.5272/jimab.2021271.3518

Received: 18/11/2019; Published online: 12/01/2021

\section{Address for correspondence:}

Maksim Zagorov

Department of Orthopaedics and Traumatology, University Hospital „St. Marina", Varna.

1, Hristo Smirnenski blvd., 9000 Varna, Bulgaria

E-mail: dr_zagorov@mail.bg 